\title{
CME Neurology
}

Edited by Dr Richard ] Davenport, consultant neurologist and honorary senior

lecturer, University of Edinburgh, UK

\section{Functional}

\section{neurological}

\section{symptoms}

\author{
Jon Stone, consultant neurologist and \\ honorary senior lecturer \\ Department of Clinical Neurosciences, \\ University of Edinburgh \\ This article has been updated from a similar \\ article published in the Journal of the Royal \\ College of Physicians of Edinburgh. ${ }^{1}$
}

\section{Introduction}

The term 'functional neurological symptoms' refers to symptoms that are not explained by disease. They may also be described as psychogenic, non-organic, somatoform, dissociative or conversion symptoms. The most common functional neurological symptoms are non-epileptic attacks and weakness, especially in emergency situations, where they may be mistaken for epilepsy or stroke. Functional symptoms often persist, are associated with distress and disability and, in the right hands, have a low rate of misdiagnosis. Physicians are often uncertain how to approach patients with such symptoms. Are they making their symptoms up? How can a diagnosis be made confidently? What is the best way to explain the diagnosis? Does treatment ever help?

This article takes you through these questions, providing practical tips for avoiding common pitfalls in diagnosis and management. No good evidence indicates that these symptoms are any more 'made up' than symptoms of irritable bowel syndrome or chronic pain. Diagnosis should be made by a neurologist on the basis of positive signs of inconsistency, such as Hoover's sign or the typical features of a non-epileptic attack. A 'functional' model of the symptoms is useful both when thinking about the problem and when explaining the diagnosis to the patient.

\section{How common?}

About one-third of neurology outpatients have symptoms that neurologists rate as only 'somewhat' or 'not at all' explained by disease. At least $20 \%$ of patients in apparent 'status epilepticus' and about one in seven patients attending a 'first fit' clinic have dissociative (non-epileptic) attacks. Patients with functional weakness are at least as common as patients with multiple sclerosis and represent the most common nonstroke diagnosis in patients wrongly given thrombolysis for presumed stroke. A recent study of 3,781 neurology patients in Scotland found that about $5 \%$ had a primary diagnosis of functional neurological symptoms, such as non-epileptic attacks, functional weakness and movement disorder. ${ }^{2,3}$ Patients also present to specialties other than neurology with visual, ${ }^{4}$ hearing and speech symptoms (typically a whispering dysphonia).

\section{Making the diagnosis}

\section{History}

Avoid making a diagnosis based on the history alone: physical examination or observation of attacks plays a key role. The diagnosis should never be made just because investigations are normal. The following features are common:

- multiple symptoms, especially pain and fatigue

- a history of other functional symptoms or syndromes, including irritable bowel and/or chronic fatigue syndrome, menorrhagia (and especially early hysterectomy), fibromyalgia or other poorly explained chronic pain syndromes; 'brittle asthma', 'atypical chest pain' and 'unexplained palpitations' are red flags for anxiety and panic

- depression or anxiety, although not all patients with functional symptoms are depressed or anxious if you want to enquire about current mood symptoms, ask if the symptoms make them feel down or worried rather than asking if they are depressed

- panic attacks: patients with nonepileptic attacks commonly have panic symptoms prior to their attacks but are usually reluctant to discuss this as they fear being told they are 'crazy';' the same is often true for patients with acute functional weakness.

Only around one-in-five patients with functional symptoms believe that stress is relevant, whereas patients with disease often think it is.

\section{Functional weakness}

Patients with functional weakness may have the following signs, although no physical sign is foolproof, especially in the acute situation:

- Hoover's sign (Fig 1): in this test, best carried out with the patient seated, weakness of hip extension returns to normal with contralateral hip flexion against resistance

- Dragging gait: patients with acute functional weakness may drag their leg behind them, with the hip externally or internally rotated (unlike patients with organic hemiparesis, who tend to circumduct the leg

Some common but less reliable signs are:

- global pattern of weakness: if someone has a left hemiparesis due to an upper motor neuron lesion then the weakness should be pyramidal in distribution that is, extensors weaker than flexors in the arm and flexors weaker than extensors in the leg; global weakness suggests functional weakness

- collapsing weakness: a limb seems to have normal power but collapses at a slight touch; pain or misunderstanding can cause false positives. 

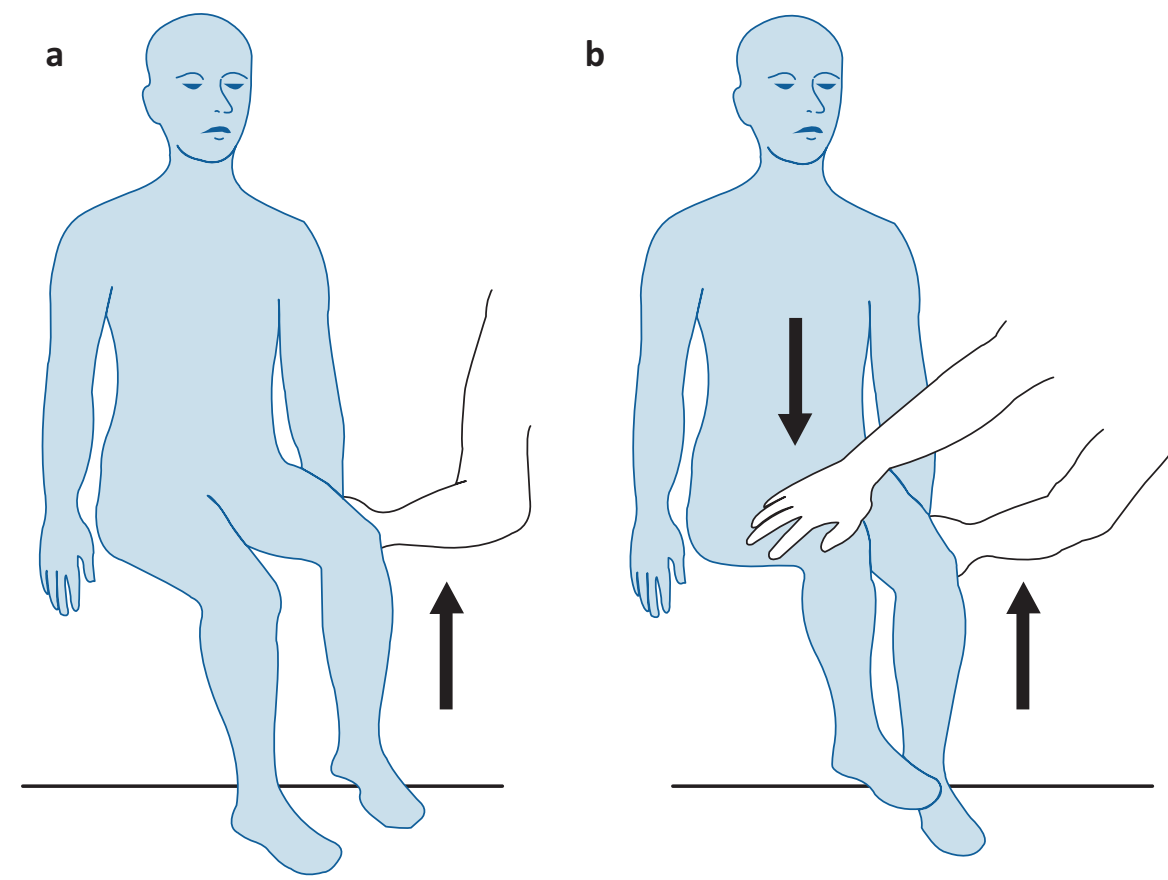

Fig 1. Hoover's sign for functional weakness - a positive physical sign. (a) hip extension is weak; (b) hip extension returns to normal with contralateral hip flexion against resistance. Reproduced with permission of RCPE. ${ }^{1}$

In an acute medicine setting, look out for the patient with multiple 'strokes' over several months, especially if they present with persistent dense weakness and a normal scan. This could be migraine but may be functional.

\section{Dissociative (non-epileptic) attacks}

The diagnosis rests on the nature of the attacks. ${ }^{6}$ A wide variety of signs have been recorded to attempt to distinguish nonepileptic attacks from epilepsy (Table 1). Ideally, the attack should be captured using video electroencephalography (EEG), but a recording from a relative's mobile phone is sometimes sufficient.

Be wary of the patient who has a seizure arising from general anaesthetic, as this is usually a non-epileptic attack. Likewise, always consider the diagnosis in anyone you are treating for 'status epilepticus', as long-duration attacks unresponsive to (or probably made worse by) diazepam are typical.

\section{Are they really ill?}

Remarkably, the presence or absence of a neurological disease does not seem to make much difference to self-rated measures of disability. In our own study of 3,781 patients, those with symptoms rated as 'not at all explained' by disease were marginally more disabled and significantly more distressed than those whose symptoms were 'completely explained' by disease. ${ }^{7}$ You might argue that these kinds of problems are best left alone as they will probably get better anyway. Unfortunately, however, virtually every follow-up study of functional neurological symptoms shows persistence of symptoms in most patients in the long term. Rates of medical retirement, consumption of health service resources and use of disability benefits problem that predominantly affects people of working age.

\section{What if the diagnosis is wrong?}

If someone is paralysed and in a wheelchair, it is reasonable to wonder whether the diagnosis of functional neurological symptoms is wrong. A systematic review of studies of misdiagnosis found that about $5 \%$ of patients had the wrong diagnosis after an average of five years. This is the same rate of misdiagnosis as for most neuindicate that this is a major public health rological and psychiatric conditions. The diagnosis is not easy though, and it is usually prudent to ask a neurologist to confirm whether it is correct. Mistakes particularly occur in the following situations: when a diagnosis is made by a non-neurologist; when too much emphasis is placed on a history of psychiatric problems; when a diagnosis is made just because something looks 'weird' (without clear evidence of inconsistency); when a patient has both functional symptoms and an underlying disease (particularly the early stages of a degenerative disease); when the patient has a gait disorder; and when the diagnosis is actually frontal lobe epilepsy.

\section{What if they're making the symptoms up?}

Many doctors ask how we can be sure that the patient is not making up their symptoms. The simple answer is that you cannot be sure. The only definitive way would be to use video surveillance or to obtain a confession, which is hardly likely to occur in routine practice. Instead, therefore, we must rely on a number of circumstantial pieces of evidence: the similarity of patients' reports of symptoms (around the world), the similarity of psychological associations such as adverse childhood experience, the persistence of symptoms in follow-up studies, the desire for investigations, the presence of wear marks in patients' shoes, and the fact that patients with non-epileptic attacks often have attacks during an EEG even when forewarned that a normal EEG means they probably do not have epilepsy. I do rarely meet patients with factitious disorder (in my NHS practice), who are consciously simulating symptoms, and I have also come across patients malingering (for money) in medicolegal practice. However, in my NHS practice I have stopped worrying about this issue. Even when I see a patient exaggerating, I ask myself whether they are doing that to convince me that there is a problem or to deceive me about a problem that is not there.

\section{Are these symptoms really 'psychogenic'?}

Most models that attempt to explain why these symptoms happen include biological, 
Table 1. Selected clinical features helpful and less helpful for distinguishing dissociative (non-epileptic) attacks from epilepsy.

\begin{tabular}{lll} 
Feature & $\begin{array}{l}\text { Dissociative } \\
\text { attacks }\end{array}$ & Epileptic seizures \\
Helpful & Common \\
Duration longer than two minutes & Common \\
Eyes and mouth closed & Common & Rare \\
Resisting eye opening & Rare & Rare \\
Visible large bite mark on side of tongue/ & Very rare \\
cheek/lip & Common & Occasional \\
Fast respiration during attack & Rare & Ceases \\
Grunting/guttural 'ictal cry' sound* & Occasional & Common \\
Weeping/upset after a seizure & Common & Very rare \\
Recall for period of unresponsiveness & Common & Very rare \\
Thrashing, violent movements & Rare & Rare \\
Post-ictal stertorous breathing & Common & Common \\
Attacks in medical situations & & Rare \\
Less helpful & Common & \\
Stereotyped attacks & Occasional & Common \\
Attack on waking from sleep & Common & Common \\
Aura & Occasional & Common \\
Urinary or faecal incontinence & Common \\
Injury & Common \\
\hline Patient report of tongue biting & Comaracteristic fragmented cry during a generalised tonic clonic seizure caused by a tonic diaphragm \\
forcing air against a tonic or clonic glottis. & & \\
\hline & & Common \\
\hline
\end{tabular}

\section{Key points}

Functional neurological symptoms are common and include functional weakness and dissociative (non-epileptic) attacks

Misdiagnosis, concern about malingering and a perception that symptoms typically resolve spontaneously are all unfounded reasons why doctors ignore these patients

Use positive evidence based on the physical examination (for example, Hoover's sign) or features of the attack to make the diagnosis but seek a neurological opinion for clarification

Explanations given to the patient by the physician should initially focus on the diagnosis and why it has been made. The doctor should resist the temptation to make a psychiatric formulation; this is not necessary for the important first steps in treatment

Not everyone benefits from treatment but more than enough patients do to make it worthwhile trying

KEY WORDS: functional, psychogenic, conversion disorder, non-epileptic attacks, dissociative psychological and interpersonal (social) factors. Adverse life experiences, including adverse experiences in childhood, and personality and emotional disorders can play a role in predisposing someone to symptoms, but not all patients have these factors. Symptoms may be triggered by a panic attack, injury or pain. Dissociative symptoms, such as depersonalisation (a feeling that your body is not part of you) or derealisation (a feeling of disconnection from the world) are often present. Beliefs about illness, especially anxiety about a serious cause, the consequences of recurrent attacks or being labelled as 'psychosomatic', often prevent effective understanding of symptoms. Vicious circles of pain, poor sleep, fatigue and disability provide further barriers to improvement. Functional imaging studies are helping us to understand the neural correlates of these symptoms and that they have a biology as well as a psychology. Ultimately, there is no 'one-sizefits-all' aetiological theory, so every patient has to be assessed individually, but perpetuating factors provide the best target for treatment.

\section{What treatment should be given?}

Doctors may both help and harm patients with functional symptoms. Giving a constructive diagnosis need not be time consuming, and successful explanation of the diagnosis can itself be highly therapeutic. A poor explanation often leads to an angered patient, who will be harder to help later on. An approach that I have outlined elsewhere ${ }^{8}$ involves the following steps:

- Explain to the patient what they do have: I personally use the terms 'functional weakness/movement disorder' and 'dissociative attacks'; use 'psychogenic' if you wish, but be aware that words like 'psychosomatic' and 'psychogenic' mean 'making it up' to most patients

- Explain why you are making the diagnosis: I show patients their Hoover's sign or discuss why their attacks can only be dissociative, which gives the diagnosis more authority and logic

- Explain what they do not have - for example, multiple sclerosis or epilepsy - and why 


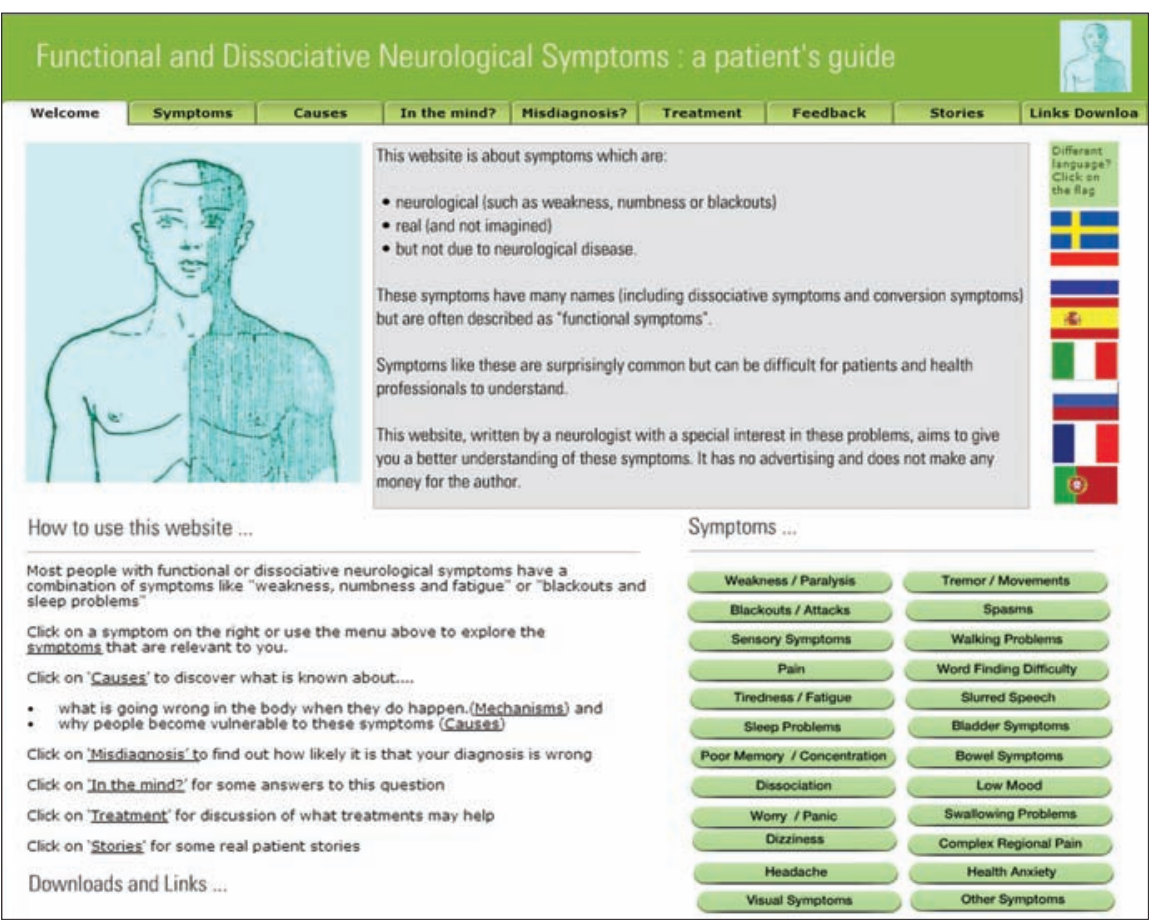

Fig 2. Free self-help websites (eg www.neurosymptoms.org, written by the author of this article) provide information for patients with functional neurological symptoms.

- Tell the patient you believe them - for example, 'I do not think you are making up or imagining these symptoms or going crazy'

- Emphasise that it is common: for example, 'I see lots of patients with similar symptoms'

- Emphasise the potential for reversibility - for example, 'Because the nervous system is not damaged, these symptoms have the potential to improve'

- Metaphors may be useful: for example, 'This is like a software problem with the brain rather than a hardware problem'

- Introduce the role of psychological factors - for example, 'This problem is not 'all in your mind, but the way that you think about things can affect it; have a think about your Hoover's sign - when you were thinking hard about moving your weak leg it did not work, but when you were distracted by moving your good leg, your weak leg moved normally'

- Use written information such as the information at www.neurosymptoms. org (a self-help website; Fig 2) and www.nonepilepticattacks.info; I also copy my clinic letters to patients

- Do not get drawn into discussions about 'why' the symptoms have happened, because it is too difficult to know why individuals have particular symptoms and this can be revisited later if necessary; the early priority is to establish confidence in the diagnosis

- Involve family and friends, as they also need to understand these symptoms.

Explanations can be very helpful for patients with these symptoms. For those with persistent symptoms, physiotherapy, encouragement, distraction techniques and graded exercise can be useful. Referral to a liaison psychiatrist may be an important step, but beware the referral to the uninterested psychiatrist who may reply 'no psychiatric disorder'. Cognitive behavioural therapy exploring patterns of thinking and behaviour over a longer period can be helpful; currently, only dissociative attacks have a clear evidence base for this kind of treatment. ${ }^{9}$ Other forms of psychotherapy may be useful but need to be conducted by someone who is familiar with functional neurological symptoms. Many patients do not improve despite treatment, so it is important to know when to stop trying. In my own practice, if I can help at least one in three patients with chronic symptoms, I regard that as a reasonable 'number needed to treat'. Little evidence is available regarding antidepressants in patients with functional neurological symptoms, but they can be used when they have symptoms known to respond to these agents, such as depression, anxiety, pain and insomnia.

\section{References}

1 Stone J. Functional neurological symptoms. J Roy Coll Phys Edinb 2011;41:38-41.

2 Stone J, Carson A, Duncan R et al. Symptoms 'unexplained by organic disease' in 1144 new neurology out-patients: how often does the diagnosis change at followup? Brain 2009;132:2878-88.

3 Edwards MJ, Bhatia KP. Functional (psychogenic) movement disorders: merging mind and brain. Lancet Neurol 2012;11:250-60.

4 Chen CS, Lee AW, Karagiannis A et al. Practical clinical approaches to functional visual loss. J Clin Neurosci 2007;14:1-7.

5 Goldstein LH, Mellers JD. Ictal symptoms of anxiety, avoidance behaviour, and dissociation in patients with dissociative seizures. $J$ Neurol Neurosurg Psychiatry 2006;77:616-21.

6 Schacter S, LaFrance Jr WC. Gates and Rowan's nonepileptic seizure, 3rd edition. Cambridge: Cambridge University Press, 2010.

7 Carson A, Stone J, Hibberd C et al. Disability, distress and unemployment in neurology outpatients with symptoms 'unexplained by organic disease'. J Neurol Neurosurg Psychiatry 2011;82:810-3.

8 Stone J. The bare essentials: functional symptoms in neurology. Pract Neurol 2009;9:179-89.

9 Goldstein LH, Chalder T, Chigwedere C et al. Cognitive-behavioral therapy for psychogenic nonepileptic seizures: a pilot RCT. Neurology 2010;74:1986-94.

\section{Address for correspondence: Dr J} Stone, Consultant Neurologist and Honorary Senior Lecturer, Department of Clinical Neurosciences, University of Edinburgh, Western General Hospital, Crewe Road, Edinburgh EH4 2XU. Email: Jon.Stone@ed.ac.uk. 